\title{
ON THE STRUCTURE OF ISOMETRICALLY EMBEDDABLE METRIC SPACES*
}

\author{
KATHLEEN NOWAK ${ }^{\dagger}$, CARLOS ORTIZ MARRERO ${ }^{\ddagger}$, AND STEPHEN J. YOUNG $\ddagger$
}

\begin{abstract}
Since its popularization in the 1970s, the Fiedler vector of a graph has become a standard tool for clustering of the vertices of the graph. Recently, Mendel and Noar, Dumitriu and Radcliffe, and Radcliffe and Williamson have introduced geometric generalizations of the Fiedler vector. Motivated by questions stemming from their work, we provide structural characterizations for when a finite metric space can be isometrically embedded in a Hilbert space.
\end{abstract}

Key words. Metric embeddings, Isometric embeddings, Hilbert spaces.

AMS subject classifications. 05C12, 30L05.

1. Introduction. Since the work of Fiedler $[11,12,13]$, the spectrum of the combinatorial Laplacian $L=T-A$, where $A$ is the adjacency matrix of a connected, simple graph $G=(V, E)$, and $T$ is the diagonal matrix of degrees, has received significant attention as a means of partitioning the vertices of the graph, see for instance [20,35]. Specifically, the smallest nonzero eigenvalue and its associated eigenvector (typically referred to as the algebraic connectivity, $a(G)$, and the Fiedler vector) have been used to create bipartitions with guaranteed quality metrics [1, 8, 31, 40, 41, 42]. More recently, the smallest nonzero eigenvalue $\lambda_{2}(G)$ of the normalized Laplacian, $\mathcal{L}=T^{-1 / 2} L T^{-1 / 2}=I-T^{-1 / 2} A T^{-1 / 2}$, and its associated eigenvector (which is sometimes also referred to as the Fiedler vector) have also been used to perform Fiedler-like clustering [33,39]. It is worth mentioning that both $a(G)$ and $\lambda_{2}(G)$ have numerous connections to structural properties of graphs beyond graph partitioning, see for instance $[5,6,9,14,32]$.

Over the past decade or so, there have been several works generalizing the ideas and quality bounds of the Fiedler partitioning schemes to multi-way partitions [2, 24, 26, 27, 33]. Generally, the idea behind these approaches has been to use the eigenvectors associated with the $k$ smallest nonzero eigenvalues to define a mapping from the vertices into $\mathbb{R}^{k}$. At this point, an approximation of a geometric clustering algorithm, such as $k$-means, is used to form the multi-way partitions. Recently, an alternative generalization of the Fiedler vector has been developed by Mendel and Noar for the combinatorial Laplacian [28, 29, 30] and subsequently by Radcliffe and Williamson [36], and Dumitriu and Radcliffe [10] for the normalized Laplacian. These generalizations are rooted in the observation that, via the Courant-Fischer theorem, there is some functional form $\mathcal{R}$ such that $a(G)$ and $\lambda_{2}(G)$ can be expressed as $\min _{f: V \rightarrow \mathbb{R}} \mathcal{R}(f)$. Furthermore, this functional form can be manipulated so that it depends only on $(f(u)-f(v))^{2}$, which is the square of the standard distance metric on $\mathbb{R}$. Thus, one can naturally defined the geometric Fiedler vector by replacing $\mathbb{R}$ with an arbitrary metric space $(X, d)$ and considering $\lambda(G, X)=\min _{f: V \rightarrow X} \mathcal{R}(f)$.

${ }^{*}$ Received by the editors on June 19, 2017. Accepted for publication on January 14, 2022. Handling Editor: Bryan Shader. Corresponding Author: Stephen J. Young

The research described in this paper was conducted under the Laboratory Directed Research and Development Program at Pacific Northwest National Laboratory, a multiprogram national laboratory operated by Battelle for the US Department of Energy. PNNL Information Release: PNNL-SA-126943

$\dagger$ Department of Homeland Security, Office of Trade (kathleen.nowak@cbp.dhs.gov). Work performed while at Pacific Northwest National Laboratory.

†Pacific Northwest National Laboratory, Richland, WA 99352 (carlos.ortizmarrero@pnnl.gov, stephen.young@pnnl.gov). 
This definition leads naturally to the question of when can the geometric Fiedler vector be thought of as an eigenvector of some linear operator. Specifically, which finite metric spaces $(X, d)$ are consistent with a definition of orthogonality, that is, they can be isometrically embedded in a Hilbert space. Although this question can be answered from an analytical point of view by the Schoenberg Embedding Theorem [37, 38], we instead approach the question from a more structural viewpoint. Specifically, for every metric $(X, d)$ associate the critical graph $G=(X, E)$ with weights given by $d$ and edge set given by $\left\{\{u, v\} \in\left(\begin{array}{c}X \\ 2\end{array}\right): \forall z \neq u, v d(u, v)<d(u, z)+d(z, v)\right\}$. In this work, we will provide a complete characterization of the unweighted critical graphs corresponding to metrics that are isometrically embeddable in a Hilbert space. Additionally, we provide structural characteristics of weighted critical graphs that are isometrically embeddable in a Hilbert space.

2. Schoenberg's spectral characterization. Although we are taking a structural point of view by identifying critically graphs corresponding to isometrically embeddable metric spaces, the analytic embedding theorem of Schoenberg will play a key role in our analysis [37, 38]. Before stating Schoenberg's result, we need to define what it means for a bivariate function on a metric space to be conditionally negative definite. To that end, let $(X, d)$ be a metric space. A function $\psi: X \times X \rightarrow \mathbb{R}$ is conditionally negative definite if for any $x_{1}, x_{2}, \ldots x_{n} \in X$ and $\alpha_{1}, \alpha_{2}, \ldots, \alpha_{n} \in \mathbb{R}$ such that $\sum_{j=1}^{n} \alpha_{j}=0$, we have

$$
\sum_{i, j=1}^{n} \alpha_{i} \alpha_{j} \psi\left(x_{i}, x_{j}\right) \leq 0 .
$$

With terminology in hand, we can now state the following theorem:

Schoenberg Embedding Theorem. Let $(X, d)$ be a metric space. There exists a Hilbert space $\mathcal{H}$ and an isometry $\phi: X \rightarrow \mathcal{H}$ if and only if $d^{2}$ is conditionally negative definite.

As we are dealing with finite metric spaces, it will be helpful to rephrase Schoenberg's result in terms of matrices. Specifically, given a finite metric space $(X, d)$ define $D$ to be the squared distance matrix for the metric, that is, $D_{x y}=d(x, y)^{2}$. From Schoenberg's result, we have that $(X, d)$ is isometrically embeddable into a Hilbert space if and only if

$$
\max _{\substack{\alpha \in \mathbb{R}^{n} \\ \alpha \perp \mathbb{1}}} \alpha^{T} D \alpha \leq 0
$$

where $\mathbb{1}$ is the all ones vector. Further, note that

$$
\max _{\substack{\alpha \in \mathbb{R}^{n} \\ \alpha \perp \mathbb{1}}} \alpha^{T} D \alpha=\max _{v \in \mathbb{R}^{n}} v^{T}\left(I-\frac{1}{n} J\right)^{T} D\left(I-\frac{1}{n} J\right) v,
$$

where $J=\mathbb{1}^{T} \mathbb{1}$ is the matrix of all ones. Hence, $(X, d)$ is isometrically embeddable if and only if $\left(I-\frac{1}{n} J\right)^{T}$ $D\left(I-\frac{1}{n} J\right)$ is negative semi-definite.

Although not necessary for our study of critical graphs, it is interesting to note that the standard proofs of Schoenberg's result for finite metric spaces, see for instance [34], are constructive. Specifically, given an arbitrary fixed point $x_{0} \in X$, the Hilbert space is given by $\mathcal{H}=\left\{\sum_{x \in X} \alpha_{x} k_{x}: \alpha_{x} \in \mathbb{R}\right\}$ with $\left\langle k_{x}, k_{y}\right\rangle=d\left(x, x_{0}\right)^{2}+d\left(y, x_{0}\right)^{2}-d(x, y)^{2}$ and the isometry is given by $\phi(x)=\frac{1}{\sqrt{2}} k_{x}$ for all $x$. In particular, the Hilbert space is isomorphic to the Hilbert space on $\mathbb{R}^{|X|}$ with the inner product given by $\alpha^{T} K \beta$ for $\alpha, \beta \in \mathbb{R}^{|X|}$ and $K_{x, y}=d\left(x, x_{0}\right)^{2}+d\left(y, x_{0}\right)^{2}-d(x, y)^{2}$. 
Since the kernel function $K$ completely determines the Hilbert space, it is worth addressing how the choice of $x_{0}$ affects $K$. To that end, consider the metric space formed by shortest path distances on $P_{3}$, the path with three vertices. When considering the three $K$ matrices generated by the vertices of $P_{3}$, we get

$$
\left[\begin{array}{lll}
0 & 0 & 0 \\
0 & 2 & 4 \\
0 & 4 & 8
\end{array}\right], \quad\left[\begin{array}{ccc}
2 & 0 & -2 \\
0 & 0 & 0 \\
-2 & 0 & 2
\end{array}\right] \text {, and }\left[\begin{array}{ccc}
8 & 4 & 0 \\
4 & 2 & 0 \\
0 & 0 & 0
\end{array}\right]
$$

The left and right kernels are clearly similar, reflecting the unique nontrivial automorphism of $P_{3}$, but one can easily see that the central kernel is not similar to the left or the right kernel by noting that the trace is 4 as compared with 10. To our knowledge, it is unknown if given generating points $x, x^{\prime} \in X$ resulting in Hilbert spaces $\mathcal{H}, \mathcal{H}^{\prime}$ and isometries $\phi, \phi^{\prime}$, there is an isometry $\psi$ between $\mathcal{H}$ and $\mathcal{H}^{\prime}$ such that $\phi^{\prime}=\psi \circ \phi$.

3. Prior work. Besides the work of Schoenberg, there is an extensive literature devoted to understanding the nature of embedding metric spaces in other spaces, typically Hilbert spaces. The work that is perhaps closest in spirit to this work is recent work toward determining the structure of (strictly) conditionally negative definite matrices [21, 22, 43]. In particular, the work of Joziak and Winkler [22, 43] is very similar to this work in spirit, in that they concern themselves with the graphical structures that result in conditionally negative definite matrices. Specifically, they consider when the matrix for the shortest path metric in a graph is conditionally negative definite. In contrast with these works, we will be focused on when the entrywise square of the distance matrix is conditionally negative definite. Somewhat surprisingly, this results in significantly different characterizations. Specifically, Joziak shows that a large class of graphs including trees and odd cycles are conditionally negative definite, and we show that almost all of these metrics are not isometrically embeddable in a Hilbert space with the only exceptions being the path and the triangle.

If, instead of requiring an isometric embedding into a Hilbert space, we allow for the distances between elements to contract we end up considering what are termed low-distortion embeddings. A survey of this broad ranging field is beyond the scope of this work, but an introduction by Indyk and Matouşek can be found in [15]. At this point, we would be remiss if we did not also mention the connection between the geometric Fiedler vector for the normalized Laplacian over the metric generated by $K_{2}$ (i.e., the two-point discrete-metric space whose elements have distance 1). Specifically, in this case, the geometric Fiedler vector gives the solution to the SPARSEST-CUT problem with pairwise unit demands. This problem is known to be NP-COMPLETE and thus can only be approximately solved efficiently. Several of these approximation schemes involve calculating an auxiliary metric via linear programming and then embedding this metric in Euclidean space with small distortion. In fact, the guarantees on the distortion often directly lead to the approximation guarantees for the algorithm. See for instance [4, 3, 7, 23, 25].

Finally, we wish to mention the work of Graham and Winkler who consider the question of whether a graph $G$ can be isometrically embedded in a graph $G^{*}$ which is the Cartesian product of simpler graphs. Although there is no universal graph $H$ such that every graph can be isometrically embedded in $H^{m}$ for some $m$, Graham and Winkler provide, given a graph $G$, a canonical means of constructing factors and a product graph $G^{*}$ such that there exists an isometric embedding of $G$ in to $G^{*}[16,17,18,19]$.

4. Structural characterizations of isometric embeddings. There is a natural correspondence between finite metric spaces and connected weighted graphs. For a connected weighted graph $G=(V, E, w)$, the shortest path distance is a metric on $V$. Conversely, given a metric space $(X, d)$, we say that a weighted 
graph $G=(V, E, w)$ represents or generates the metric if there exists a bijection $\phi: X \rightarrow V$ such that for all $x, x^{\prime} \in X, d_{G}\left(\phi(x), \phi\left(x^{\prime}\right)\right)=d_{X}\left(x, x^{\prime}\right)$. For example, $\left(X,\left(\begin{array}{c}X \\ 2\end{array}\right), d\right)$ generates the metric space $(X, d)$. However, this graph representation is not unique. One can often remove edges from this graph without altering the metric. We will show, however, that there is a unique minimal graph representing the metric and that this graph is the critical graph for the metric.

Proposition 4.1. For any finite metric space, the associated critical graph is the unique graph with the minimum number of edges that generates the metric.

Proof. Let $(X, d)$ be a finite metric space and let $G=(X, E, w)$ be the critical graph that is associated with the metric. First, we will show that $G$ generates the metric $(X, d)$. To that end, let $d^{G}(\cdot, \cdot)$ be the metric on $X$ induced by $G$. Since $d^{G}$ is the shortest path metric on $G$, we have that for any $u \neq v$, there exists a path $u=x_{0}, x_{1}, \ldots, x_{t+1}=v$ in $G$ such that

$$
d^{G}(u, v)=\sum_{i=0}^{t} w\left(x_{i}, x_{i+1}\right)=\sum_{i=0}^{t} d\left(x_{i}, x_{i+1}\right) \geq d(u, v)
$$

Thus, $d^{G}$ is an upper bound on $d$. Suppose now that $d \neq d^{G}$, then the set $\left\{\{u, v\}: d(u, v)<d^{G}(u, v)\right\}$ is non-empty and hence has an element $(x, y)$ that minimizes $d(x, y)$. Now since $d(x, y)<d^{G}(x, y)$, we know that $x \not y$ in $G$, and thus there is some other element $z \in X$ such that $d(x, y)=d(x, z)+d(z, y)$. But by the construction of $\{x, y\}, d(x, z)=d^{G}(x, z)$ and $d(z, y)=d^{G}(z, y)$, and hence $d(x, y)<d^{G}(x, y) \leq$ $d^{G}(x, z)+d^{G}(z, y)=d(x, z)+d(z, y)=d(x, y)$, a contradiction.

To complete the proof, it suffices to show that every edge in the critical graph $G$ must be present in every graph that generates the metric space $(X, d)$. However, this is obvious as if $\{x, y\} \in E$ then $d(x, y)<d(x, z)+d(z, y)$ for every $z$ other than $x$ or $y$, and thus the metric cannot be recovered for $\{x, y\}$ via any nontrivial path.

From the discussion above, it suffices to view a finite metric space as a weighted graph adorned with the shortest path distance. Let $H$ be a weighted graph metric with squared distance matrix $D$. Then, as remarked above, $H$ can be isometrically embedded into a Hilbert space if and only if

$$
\min _{\substack{\alpha \in \mathbb{R}^{n} \\ \alpha \perp \mathbb{1}}} \alpha^{T} D \alpha \leq 0
$$

Next, we characterize the weighted graphs that satisfy this criterion.

4.1. Unweighted graphs. We first consider the case where the weights in the critical graph are all the same, that is, where the critical graph may be viewed as an unweighted graph.

ThEOREM 4.2. Let $H=(V, E, w)$ be a weighted graph with weight function $w=\mathbb{1}$. Then, $H$ can be isometrically embedded in a Hilbert space if and only if $H$ is a path or a complete graph.

Proof. We start by analyzing the subgraph induced by the closed neighborhood of a vertex.

Claim 1. If $\min _{\substack{\alpha \in \mathbb{R}^{n} \\ \alpha \perp \mathbb{1}}} \alpha^{T} D \alpha \leq 0$, then for any vertex $v$ with $d_{v}>2$, the closed neighborhood, $N[v]$, is complete.

Proof. Suppose that $H$ contains a vertex $v$ of degree at least three such that $N[v] \neq K_{d_{v}+1}$. Let $u$ and $w$ be nonadjacent neighbors of $v$ and let $z$ be a third neighbor. Note that the graph $H^{\prime}$ induced by $\{v, u, z, w\}$ is a shortest path subgraph of $H$. Further, $H^{\prime}$ must be one of the graphs shown in Figure 1 with corresponding 


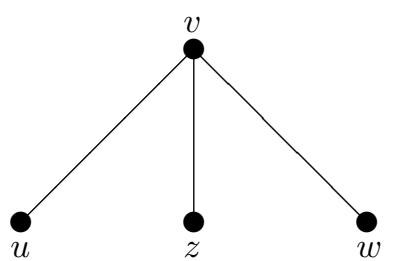

$$
\left[\begin{array}{llll}
0 & 1 & 1 & 1 \\
1 & 0 & 4 & 4 \\
1 & 4 & 0 & 4 \\
1 & 4 & 4 & 0
\end{array}\right]
$$

(a)

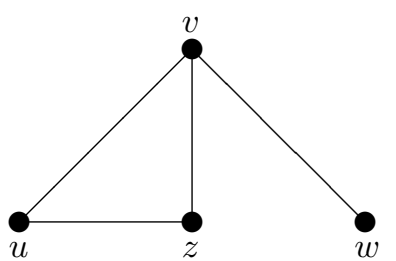

$$
\left[\begin{array}{llll}
0 & 1 & 1 & 1 \\
1 & 0 & 1 & 4 \\
1 & 1 & 0 & 4 \\
1 & 4 & 4 & 0
\end{array}\right]
$$

(b)

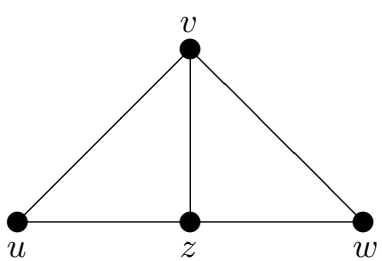

$$
\left[\begin{array}{llll}
0 & 1 & 1 & 1 \\
1 & 0 & 1 & 4 \\
1 & 1 & 0 & 1 \\
1 & 4 & 1 & 0
\end{array}\right]
$$

(c)

Figure 1. Possible Neighborhood Configurations and Associated Principal Submatrices

submatrix of $D$. In each case, there exists a vector $\alpha$ such that $\alpha^{T} D \alpha>0$. For example, if the vertices are ordered starting with $v, u, w, z$, then assigning $\alpha$ to be $(-3,1,1,1,0, \ldots, 0),(4,-1,-1,-2,0, \ldots 0)$, and $(1,-1,1,-1,0, \ldots, 0)$ for configurations $(a),(b)$, and $(c)$, respectively, yields $\alpha^{T} D \alpha>0$.

Now suppose that we can isometrically embed $H$ in a Hilbert space. Then by the previous claim, $H$ must be a cycle, path, or complete graph. Next, we show that $H$ is not a cycle of length greater than 3 .

Claim 2. If $\min _{\substack{\alpha \in \mathbb{R}^{n} \\ \alpha \perp \mathbb{1}}} \alpha^{T} D \alpha \leq 0$, then $H \neq C_{n}$ for $n \geq 4$.

Proof. Suppose that $H=C_{n}$ for some $n \geq 4$. If $n=2 k$ is even, then by labeling the vertices sequentially (see Figure 2) and ordering them starting with $0,2 k-1, k-1, k, D$ has the following principal submatrix:

$$
\left[\begin{array}{cccc}
0 & 1 & (k-1)^{2} & k^{2} \\
1 & 0 & k^{2} & (k-1)^{2} \\
(k-1)^{2} & k^{2} & 0 & 1 \\
k^{2} & (k-1)^{2} & 1 & 0
\end{array}\right]
$$

Then setting $\alpha=(1,-1,-1,1,0, \ldots, 0), \alpha^{T} D \alpha=8(k-1)>0$. If $n=2 k+1$ is odd, then labeling the vertices sequentially (see Figure 2) and ordering them starting with $0,1, k, k+1, D$ has the following principal submatrix:

$$
\left[\begin{array}{cccc}
0 & 1 & k^{2} & k^{2} \\
1 & 0 & (k-1)^{2} & k^{2} \\
k^{2} & (k-1)^{2} & 0 & 1 \\
k^{2} & k^{2} & 1 & 0
\end{array}\right]
$$

Then setting $\alpha=(1,-1,1,-1,0, \ldots, 0), \alpha^{T} D \alpha=2(2 k-3)>0$.

Finally, we show that if $H$ is a path or complete graph then $D$ is conditionally negative definite. First, suppose that $H=K_{n}$ and consider $\alpha \in \mathbb{R}^{n}$ such that $\alpha \perp \mathbb{1}$. Then, $D=A=J-I, \alpha=$ $\left(\alpha_{1}, \alpha_{2}, \ldots, \alpha_{n-1},-\sum_{i=1}^{n-1} \alpha_{i}\right)$, and $\alpha^{T} D \alpha=-\langle\alpha, \alpha\rangle \leq 0$. Now suppose that $H=P_{n}$. By our previous observation, it suffices to show that $M:=\left(I-\frac{1}{n} J\right) D\left(I-\frac{1}{n} J\right)$ is negative semi-definite. Now, $D_{i j}=(i-j)^{2}$ 


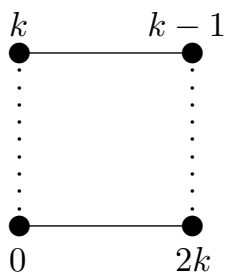

(a) Even Cycle

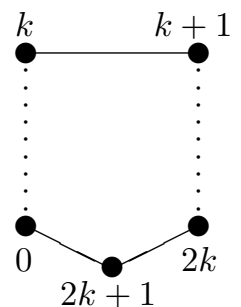

(b) Odd Cycle

Figure 2. Cycle Labelings

$$
\begin{aligned}
M_{i j} & =(i-j)^{2}-\frac{1}{n} \sum_{k=1}^{n}(k-j)^{2}-\frac{1}{n} \sum_{\ell=1} n(\ell-i)^{2}+\frac{1}{n^{2}} \sum_{k=1}^{n} \sum_{\ell=1}^{n}(k-\ell)^{2} \\
& =\left(i^{2}-2 i j+j^{2}\right)-\frac{1}{n}\left(n\left(i^{2}+j^{2}\right)-2 \sum_{k=1}^{n} k(i+j)+2 \sum_{k=1}^{n} k^{2}\right)+\frac{1}{n^{2}} \sum_{k=1}^{n} \sum_{\ell=1}^{n}\left(k^{2}-2 k \ell+\ell^{2}\right) \\
& =-2 i j+\frac{2}{n}\left(\begin{array}{c}
n+1 \\
2
\end{array}\right)(i+j)-\frac{2}{n^{2}}\left(\begin{array}{c}
n+1 \\
2
\end{array}\right)\left(\begin{array}{c}
n+1 \\
2
\end{array}\right) \\
& =-2\left(i-\frac{1}{n}\left(\begin{array}{c}
n+1 \\
2
\end{array}\right)\right)\left(j-\frac{1}{n}\left(\begin{array}{c}
n+1 \\
2
\end{array}\right)\right) .
\end{aligned}
$$

Thus, $M=-2 v v^{T}$ where $v_{i}=\left(i-\frac{1}{n}\left(\begin{array}{c}n+1 \\ 2\end{array}\right)\right)$ so $M$ is negative semi-definite as desired.

4.2. Weighted graphs. The goal of this section is to describe the structure of isometrically embeddable metric spaces when the associated critical graphs are allowed to have edge weights other than 1 . Specifically, we will provide a complete characterization of all two-connected critical graphs that have a weighting which admits an isometric embedding of the associated metric space. By simple enumeration, it is easy to see that all connected graphs on at most three vertices admit a weighting so that the associated metric may be isometrically embedding in a Hilbert space. Thus, to begin our characterization, we provide a complete characterization of critical graphs on four vertices that admit an isometrically embeddable weighting.

LEMMA 4.3. Let $(X, d)$ be a metric space with four elements that can be isometrically embedded into a Hilbert space. Let $G=(X, E, w)$ be the critical graph associated with $(X, d)$, then $G$ is either a path, $K_{4}$, or $K_{4}-e$.

Proof. Denote the elements of $X$ by $x_{1}, x_{2}, x_{3}, x_{4}$ and suppose that $G$ is not complete. Enumeration of the connected, non-complete graphs on four vertices shows that $G$ has is a vertex, say $x_{3}$, with two nonadjacent neighbors neighbors, say $x_{2}$ and $x_{4}$. Further, by the triangle inequality, we may also assume that $d\left(x_{2}, x_{4}\right)=d\left(x_{2}, x_{3}\right)+d\left(x_{3}, x_{4}\right)$. For simplicity of notation, denote $d\left(x_{i}, x_{j}\right)$ by $d_{i j}$. Now by Schoenberg's Embedding Theorem, for any choice of $\alpha_{1}, \alpha_{2}, \alpha_{3} \in \mathbb{R}$, the vector $\alpha=\left(\alpha_{1}, \alpha_{2}, \alpha_{3},-\alpha_{1}-\alpha_{2}-\alpha_{3}\right)^{T}$ satisfies $\alpha^{T} D \alpha \leq 0$ where $D_{i j}=d_{i j}^{2}$. We now note that

$$
\alpha^{T} D \alpha=2\left[-\sum_{i=1}^{3} d_{i 4}^{2} \alpha_{i}^{2}+\sum_{1 \leq i<j \leq 3}\left(d_{i j}^{2}-d_{i 4}^{2}-d_{j 4}^{2}\right) \alpha_{i} \alpha_{j}\right]
$$




$$
\begin{aligned}
& =2\left[-\left(\sum_{i=1}^{3} d_{i 4} \alpha_{i}\right)^{2}+\sum_{1 \leq i<j \leq 3}\left(d_{i j}^{2}-\left(d_{i 4}-d_{j 4}\right)^{2}\right) \alpha_{i} \alpha_{j}\right] \\
& =2\left[-\left(\sum_{i=1}^{3} d_{i 4} \alpha_{i}\right)^{2}+\left(d_{12}^{2}-\left(d_{14}-d_{24}\right)^{2}\right) \alpha_{1} \alpha_{2}+\left(d_{13}^{2}-\left(d_{14}-d_{34}\right)^{2}\right) \alpha_{1} \alpha_{3}\right],
\end{aligned}
$$

where the last equality comes from the fact that $d_{24}=d_{23}+d_{34}$. Thus, if $\alpha_{1}$ is chosen to be $-d_{24} \alpha_{2}-d_{34} \alpha_{3} / d_{14}$, we have that

$$
\alpha^{T} D \alpha=2 \alpha_{1}\left(\left(d_{12}^{2}-\left(d_{14}-d_{24}\right)^{2}\right) \alpha_{2}+\left(d_{13}^{2}-\left(d_{14}-d_{34}\right)^{2}\right) \alpha_{3}\right) .
$$

Letting

$$
u=\left[\begin{array}{l}
d_{24} \\
d_{34}
\end{array}\right], \quad v=\left[\begin{array}{l}
d_{12}^{2}-\left(d_{14}-d_{24}\right)^{2} \\
d_{13}^{2}-\left(d_{14}-d_{34}\right)^{2}
\end{array}\right], \quad \text { and } w=\left[\begin{array}{l}
\alpha_{2} \\
\alpha_{3}
\end{array}\right]
$$

we have that

$$
\alpha^{T} D \alpha=-\frac{2}{d_{14}}\langle u, w\rangle\langle v, w\rangle .
$$

Since the choice of $w$ is arbitrary in $\mathbb{R}^{2}$, there exists some nonnegative constant $c$ such that $v=c u$, as otherwise there is a some $w$ such that $\langle u, w\rangle\langle v, w\rangle<0$.

We now consider the shortest path between $x_{1}$ and $x_{4}$ in the critical graph $G$ of $(X, d)$. This path is either $\left(x_{1}, x_{2}, x_{3}, x_{4}\right),\left(x_{1}, x_{3}, x_{4}\right)$, or $\left(x_{1}, x_{4}\right)$. In the first case $G$ is a path, so consider the case where the shortest path is $x_{1}, x_{3}, x_{4}$. In this case, we have that $d_{14}=d_{13}+d_{34}$, and $d_{13}<d_{12}+d_{23}$. Substituting the value for $d_{14}$ into the equation for $v$, we have

$$
v=\left[\begin{array}{l}
d_{12}^{2}-\left(d_{13}+d_{34}-d_{24}\right)^{2} \\
d_{13}^{2}-\left(d_{13}+d_{34}-d_{34}\right)^{2}
\end{array}\right]=\left[\begin{array}{c}
d_{12}^{2}-\left(d_{13}-d_{23}\right)^{2} \\
0
\end{array}\right] .
$$

But, as $d_{13}<d_{12}+d_{23}$ and $d_{23}<d_{13}+d_{12}$, we have that the first component of $v$ is nonzero. Thus, as both components of $u$ are strictly positive, there is no nonnegative constant $c$ such that $v=c u$, a contradiction.

At this point, we may assume that the critical graph contains the edge $\left\{x_{1}, x_{4}\right\}$. But then we have that $d_{14}<d_{13}+d_{34}$ and $d_{34}<d_{14}+d_{13}$ and so the second component of $v$ is strictly positive. By the same argument as above, this implies that the first component of $v$ is strictly positive, and in particular that $d_{12}>\left|d_{14}-d_{24}\right|$. Thus, the required constant $c$ is strictly positive. Now the existence of such a constant is equivalent to $v_{2} u_{1}-v_{1} u_{2}=0$. Combining this with the fact that $d_{24}=d_{23}+d_{34}$, we get that

$$
0=v_{2} u_{1}-v_{1} u_{2}=d_{23} d_{13}^{2}-d_{23} d_{14}^{2}+d_{34} d_{13}^{2}-d_{34} d_{12}^{2}+d_{34} d_{23}^{2}+d_{23} d_{34}^{2} .
$$

At this point, we may consider the shortest path between $x_{1}$ and $x_{2}$ in the critical graph of $(X, d)$. As above, there are two non-path choices, namely, $x_{1}, x_{2}$ and $x_{1}, x_{3}, x_{2}$. First, assume that the shortest path is $x_{1}, x_{3}, x_{2}$ and hence $d_{12}=d_{13}+d_{23}$. Substituting into Equation 4.1 , we have that

$$
\begin{aligned}
0 & =d_{23} d_{13}^{2}-d_{23} d_{14}^{2}+d_{34} d_{13}^{2}-d_{34}\left(d_{13}+d_{23}\right)^{2}+d_{34} d_{23}^{2}+d_{23} d_{34}^{2} \\
& =d_{23} d_{13}^{2}-d_{23} d_{14}^{2}-2 d_{34} d_{13} d_{23}+d_{23} d_{34}^{2} \\
& =d_{23}\left[d_{13}^{2}-d_{14}^{2}-2 d_{34} d_{13}+d_{34}^{2}\right] \\
& =d_{23}\left[\left(d_{13}-d_{34}\right)^{2}-d_{14}^{2}\right] .
\end{aligned}
$$


But this implies that $\left|d_{13}-d_{34}\right|=d_{14}$ and hence, either $d_{13}=d_{14}+d_{34}$ and $x_{1} \nsim x_{3}$ or $d_{34}=d_{13}+d_{14}$ and $x_{3} \nsim x_{4}$. Both cases contradict our assumptions, so we may assume that $x_{1} \sim x_{2}$ in the critical graph.

We now consider whether $x_{1}$ and $x_{3}$ are adjacent in the critical graph. If $x_{1} \nsim x_{3}$, then either $d_{13}=$ $d_{12}+d_{23}$ or $d_{13}=d_{14}+d_{34}$. Substituting into equation 4.1 , we get

$$
0=d_{23}\left[\left(d_{12}+d_{23}+d_{34}\right)^{2}-d_{14}^{2}\right] \text {, }
$$

and

$$
0=d_{34}\left[\left(d_{34}+d_{14}\right)^{2}+d_{23}^{2}+2 d_{14} d_{34}-d_{14}^{2}\right]
$$

respectively. Both of these equations are obviously false, yielding $x_{1} \sim x_{3}$. Thus, we have that the critical graph of $(X, d)$ is either a path, $K_{4}$, or $K_{4}-e$.

It is relatively easy to construct infinite families of distinct metrics with integer distances whose critical graphs are $P_{4}, K_{4}$, or $K_{4}-e$ and are embeddable into a Hilbert space. For instance, for $K_{4}-e$ we have

OBSERVATION 4.4. There exists an infinite family of isometrically embeddable metric spaces $(X, d)$ on four elements such that the critical graph for $(X, d)$ is $K_{4}-e$ and $d$ is integer-valued. Further, each metric can be isometrically embedded in $\mathbb{R}^{2}$.

Proof. Fix $z \in \mathbb{N}$ and $\left(p_{i}, q_{i}\right)$ for $i=1,2,3$ such that $p_{i} q_{i}=z$ and $p_{i} \equiv q_{i}(\bmod 2)$. Define $x_{i}=$ $\left(\frac{p_{i}^{2}-q_{i}^{2}}{2}, 0\right)$ for $i=1,2,3$, and $x_{4}=(0, z)$. Then, since $\left(p_{i} q_{i}, \frac{p_{i}^{2}-q_{i}^{2}}{2}, \frac{p_{i}^{2}+q_{i}^{2}}{2}\right)$ are three integers that form a Pythagorean triple for $i=1,2,3$, the submetric space of $\mathbb{R}^{2}$ on these four points has $K_{4}-e$ as a critical graph and integer weights as desired.

We can use the complete characterization of four-element embeddable metrics to show that paths are the unique class of 1-connected, weighted. isometrically embeddable, critical graphs.

LEMMA 4.5. Let $(X, d)$ be a metric space that can be isometrically embedded into a Hilbert space and let $G=(X, E, d)$ be the critical graph associated with $(X, d) . G$ is either a path or 2-connected and further, if $\{u, v\}$ is a vertex cut, then $u \sim v$.

Proof. Suppose that $G$ contains a cut vertex. If every cut vertex has degree 2, $G$ is a path. Thus, suppose that $G$ contains a cut vertex $v$ of degree at least 3. Let $x, y$, and $z$ be neighbors of $v$ such $z$ belongs to a different component of $G \backslash v$ than both $x$ and $y$. Then $x, v, z$ and $y, v, z$ are shortest paths in $G$. Thus, the critical graph of the submetric space on $\{v, x, y, z\}$ is either the claw or the claw with a single additional edge, both of which are forbidden. Thus, $G$ is either a path or 2-connected.

Now suppose that $G$ is strictly 2 -connected and let $\{u, v\}$ be a cut set such that $u \neq v$. Let $C_{1}, C_{2}, \ldots, C_{k}$ denote the components of $G \backslash\{u, v\}$ and let $H_{i}:=G\left[\{u, v\} \cup V\left(C_{i}\right)\right]$. Note that since $G$ has no cut vertices each $H_{i}$ has a shortest path from $u$ to $v$, say $P_{i}$. One of these shortest paths must be the shortest path in $G$, say $P_{1}$ in $H_{1}$. Since $u \not v, P_{1}$ and $P_{2}$ contain vertices $x$ and $y$, respectively, different from $u$ and $v$.

Now consider the critical graph $G^{\prime}$ of the submetric on $\{u, v, x, y\}$. Since $P_{1}$ is a shortest path in $G$, $d(u, x)+d(x, v)=d(u, v)$ and so the edge $\{u, v\}$ is not present in $G^{\prime}$. Further, since no shortest path from $u$ or $v$ to $x$ in $G$ contains $y, u, x, v$ is a path in $G^{\prime}$. Next, observe that a shortest path from $x$ to $y$ in $G$ must go through either $u$ or $v$. Thus, the edge $\{x, y\}$ is not present in $G^{\prime}$. Now since $G^{\prime}$ is connected, $y$ is adjacent to at least one of $u$ and $v$. Without loss of generality, assume that the edge $\{y, u\}$ is present. If the edge $\{y, v\}$ is also present, we are done since the critical graph of $G^{\prime}$ is $C_{4}$, a contradiction. 


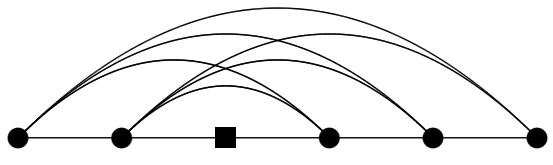

(a) $k=3$

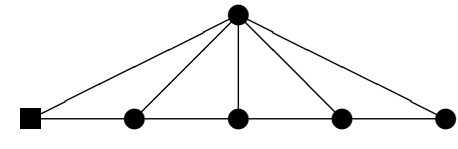

(b) $k=2$

Figure 3. 6 Vertex Critical Graphs with Isometric Embeddings by Theorem 4.6.

Thus, suppose that $\{y, v\}$ is not present. Then $d(y, v)=d(y, u)+d(u, v)$, so $P_{2}$ is not a shortest path in $G$. Further, there exists a vertex $y^{\prime}$ adjacent to $y$ on $P_{2}$ between $y$ and $v$. Now starting from $y$ and moving along $P_{2}$, we eventually find adjacent vertices $z_{u}$ and $z_{v}$ such that a shortest path from $z_{u}$ to $v$ goes through $u$ and a shortest path from $z_{v}$ to $v$ does not. Now consider the critical graph $G^{\prime \prime}$ of the submetric on $\left\{u, v, z_{u}, z_{v}\right\}$. Since $P_{2}$ is not a shortest path in $G$, the edge $\{u, v\}$ must be present. Additionally, the edge $\left\{z_{u}, z_{v}\right\}$ is present as $z_{u}$ and $z_{v}$ are adjacent in $G$. Then since a shortest path from $z_{v}$ to $u$ in $G$ must go through either $z_{u}$ or $v$, we see that the edge $\left\{z_{v}, u\right\}$ is not present. In the same vain, we also have that the edge $\left\{z_{u}, v\right\}$ is not present. Finally, by the selection of $z_{u}$ and $z_{v}$, the edges $\left\{z_{u}, u\right\}$ and $\left\{z_{v}, v\right\}$ are present. Thus, we find that $G^{\prime \prime}$ is $C_{4}$, a contradiction.

Finally, we complete the characterization of isometrically embeddable 2-connected critical graphs by providing a complete listing of 2-connected critical graphs that have an isometric embedding.

TheOREm 4.6. Let $(X, d)$ be a metric space that can be isometrically embedded into a Hilbert space. Let $G=(X, E, w)$ be the critical graph associated with $(X, d)$. If $G$ is 2-connected, but not 3-connected, then $G$ contains a Hamiltonian path $v_{1}, \ldots, v_{|X|}$ and further, there is some $2 \leq k \leq|X|-1$ such that $v_{i} \sim v_{j}$ if and only if $|i-j|=1$ or $i<k<j$.

Proof. Assume $G$ is 2-connected and not 3-connected, then there exists some pair of elements $\{u, v\}$ such that $G-\{u, v\}$ is disconnected. Furthermore, from Lemma 4.5, we know that $u \sim v$. We first show that $G-\{u, v\}$ has precisely two components. Suppose this is not the case: let $x, y, z$ be in distinct components. Now consider the shortest paths between these elements, pairwise. Since $\{u, v\}$ is a cut and the vertices are in separate components, each such shortest path must pass through either $u$ or $v$. By the pigeonhole principle, one of $u$ or $v$ has at least two shortest paths passing through it. Without loss of generality, suppose this is $u$ and the two shortest paths passing through it are between $x$ and $y$ as well as between $x$ and $z$. Now consider the submetric induced by $\{x, y, z, u\}$. Since $x, y, z$ are all in different components, vertex $u$ has degree 3 in the critical graph associated with this metric. Furthermore, since the shortest path between $x$ and either $y$ or $z$ passes through $u$, the edges $\{x, y\}$ and $x, z$ are not present in the critical graph. Thus, the associated critical graph is either a claw or a claw plus an edge, neither of which are isometrically embeddable by Lemma 4.3 . Hence, $G-\{u, v\}$ has precisely two components.

Now for any $x \in X$ other than $u$ or $v$, define $V_{x}$ as the set of elements $w$ in $X$ such that a shortest path between $x$ and $w$ passes through $v$ and that $w$ and $x$ are in different components of $G-\{u, v\}$. Define $U_{x}$ analogously. We note that if $a, b \in V_{x}$, then the critical graph associated with the submetric induced by $\{v, x, a, b\}$ has no edge from $x$ to either $a$ or $b$. Thus, since the entire metric is isometrically embeddable, the associated critical graph must be a path. Furthermore, this implies that the elements of $V_{x}$ are totally ordered by distance to $x, G\left[V_{x}\right]$ is a path, and for any two vertices in $V_{x}$ there exists a shortest path between them that is entirely contained in $G\left[V_{x}\right]$. 
Now suppose there exists some $a \in V_{x} \cap U_{x}$ and consider the induced metric formed by $\{x, u, v, a\}$. Note that we may rescale the distances in the submetric so that $d(x, a)=1$ without affecting the isometric embedability of the submetric. In this case, letting $d(x, u)=\epsilon, d(x, v)=\delta$, and $d(u, v)=\gamma$ we have that

$$
D=\left[\begin{array}{cccc}
0 & 1 & \delta^{2} & \epsilon^{2} \\
1 & 0 & (1-\delta)^{2} & (1-\epsilon)^{2} \\
\delta^{2} & (1-\delta)^{2} & 0 & \gamma^{2} \\
\epsilon^{2} & (1-\epsilon)^{2} & \gamma^{2} & 0
\end{array}\right]
$$

up to rearrangment of the columns. If we consider $\alpha$ of the form $[1,-\zeta,-1, \zeta]$, then we have that

$$
\begin{aligned}
\alpha^{T} D \alpha & =-2\left(1+\gamma^{2}\right) \zeta+2\left(\zeta \epsilon^{2}+\zeta(1-\delta)^{2}-\delta^{2}-\zeta^{2}(1-\epsilon)^{2}\right) \\
& =-2\left(\delta^{2}-\left(1+\gamma^{2}-\epsilon^{2}-(1-\delta)^{2}\right) \zeta+(1-\epsilon)^{2} \zeta^{2}\right) .
\end{aligned}
$$

Viewing $\alpha^{T} D \alpha$ as a function of $\zeta$, we have that the discriminant is

$$
\left(1+\gamma^{2}-\epsilon^{2}-(1-\delta)^{2}\right)^{2}-4 \delta^{2}(1-\epsilon)^{2},
$$

which is strictly positive so long as $1+\gamma^{2}-\epsilon^{2}-(1-\delta)^{2}-2 \delta(1-\epsilon)>0$. Observing that by the triangle inequality, $\gamma^{2}>(\delta-\epsilon)^{2}$, we have that

$$
\begin{aligned}
1+\gamma^{2}-\epsilon^{2}-(1-\delta)^{2}-2 \delta(1-\epsilon) & >1+(\delta-\epsilon)^{2}-\epsilon^{2}-(1-\delta)^{2}-2 \delta(1-\epsilon) \\
& =1+\delta^{2}-2 \delta \epsilon+\epsilon^{2}-\epsilon^{2}-1+2 \delta-\delta^{2}-2 \delta+2 \delta \epsilon \\
& =0 .
\end{aligned}
$$

Thus, the discriminate is strictly positive and so there exists some choice of $\zeta$ such that $\alpha^{T} D \alpha>0$, contradicting the embedability of $(X, d)$. As a consequence, we have that $V_{x} \cap U_{x}=\varnothing$ for all $x \in X-\{u, v\}$. In particular, if $a$ and $b$ are in different components of $G-\{u, v\}$, then there is precisely one of $\{u, v\}$ that lies on all of the shortest paths between $a$ and $b$.

Suppose there exists some pair $a \in U_{x} \cup\{u\}$ and $b \in V_{x} \cup\{v\}$ such that $a \not b$. Let $w$ be a vertex on a shortest path between $a$ and $b$ and consider the submetric determined by $\{a, b, w, x\}$. Without loss of generality, assume that $w$ lies in $U_{x} \cup\{u\}$. Since the distances to $u$ in $U_{x} \cup\{u\}$ can be totally ordered, this implies that either $w$ is closer to $u$ than $a$ or $w$ is farther from $u$ than $a$. In the former case, we have that the critical graph is a claw plus an edge and is not isometrically embeddable in a Hilbert space. In the latter case, recalling that $U_{x} \cap V_{x}=\varnothing$ we have that the critical graph is $C_{4}$ and hence is not isometrically embeddable in a Hilbert space. Thus, if $x$ is a vertex adjacent to both $u$ and $v$, there is a path $V_{x}, v, x, u, U_{x}$ and every element in $V_{x} \cup\{v\}$ is adjacent to every element in $U_{x} \cup\{u\}$. As a consequence, to complete the proof it suffices to show that there exists some 2-cut in $G$ such that there is a component containing precisely one vertex.

To that end, suppose that $V_{x}=\varnothing$ and let $u=u_{0}, u_{1}, u_{2}, \ldots, u_{t}$ be the ordering of elements of $U_{x} \cup\{u\}$ in terms of increasing distance to $u$. Now note that the only neighbors of $u_{t}$ are $u_{t-1}$ and $v$ and hence $\left\{u_{t-1}, v\right\}$ is a 2-cut with a component having precisely one element. By applying an analogous argument in the case $U_{x}=\varnothing$, we may assume without loss of generality that for any vertex $x \in X-\{u, v\}$, both $U_{x}$ and $V_{x}$ are non-empty.

Now choose some $a \in U_{x}$ and $b \in V_{x}$ and consider $V_{a}$. Since $U_{a}$ and $V_{a}$ are disjoint, $x \notin V_{a}$, and thus there exists some $z \in V_{a}$ such that $z \neq x$. Now suppose that the shortest paths between $b$ and $z$ goes through 


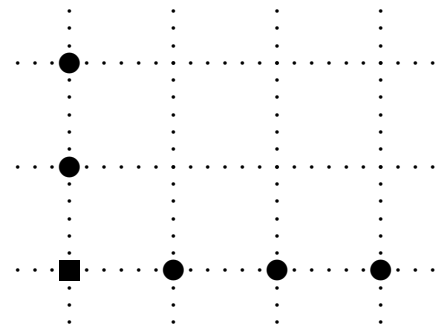

(a) Embedding Yielding Figure 3a

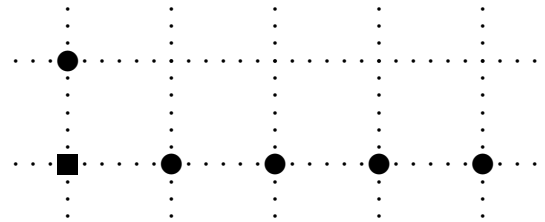

(b) Embedding Yielding Figure 3b

Figure 4. Embeddings in $\mathbb{R}^{2}$ of Metrics with Critical Graphs Given in Figure 3.

$v$ and consider the submetric induced by $\{v, x, b, z\}$. As all shortest paths between $x$ and $b$, as well as $z$ and $b$ go through $v$, the associated critical graph has $v$ as vertex of degree 3 and no edges between $\{x, z\}$ and $b$. Thus, the associated critical graph is either a claw or a claw plus an edge, and hence is not isometrically embeddable in a Hilbert space. Hence, we have that the shortest paths between $b$ and $z$ pass through $u$. Now, by the fact that all shortest paths between vertices in opposite components pass through precisely one of $u$ and $v$, we have that

$$
\begin{gathered}
d(x, u)+d(u, a)<d(x, v)+d(v, a) \\
d(x, v)+d(v, b)<d(x, u)+d(u, b) \\
d(z, u)+d(u, b)<d(z, v)+d(v, b) \\
d(z, v)+d(v, a)<d(z, u)+d(u, a) .
\end{gathered}
$$

Summing these inequalities and canceling we get $0<0$, a contradiction. Thus, there exists some vertex $x$ such that one of $U_{x}$ and $V_{x}$ is empty, completing the proof.

It is easy to find weightings for the class graphs described in Theorem 4.6 so that it is the critical graph of a metric space which is isometrically embeddable in a Hilbert space. Specifically, fix some pair of positive integers, $k$ and $n$, such that $2 \leq k \leq n$ and let $S_{n, k}=\{(0,0)\} \cup\{(i, 0): 1 \leq i<k\} \cup\{(0, j): 1 \leq n-k\} \subseteq \mathbb{N}^{2}$. Consider the critical graph induced by $(S, d)$ where $d$ is the standard distance metric on $\mathbb{N}^{2}$. The critical graph $G$ for this metric consists of a path $(k-1,0),(k-2,0), \ldots,(0,0),(0,1),(0,2), \ldots,(0, n-k)$ as well as edges between any pair of points $(x, 0)$ and $(0, y)$ where $x, y \neq 0$. That is, $G$ has a Hamiltonian path with an identified non-endpoint vertex, $(0,0)$, and every pair of vertices on opposite sides of $(0,0)$ are connected by an edge. Observing that $\mathbb{N}^{2}$ sits isometrically in the standard Hilbert space on $\mathbb{R}^{2}$ completes the construction.

5. Geometric spectrum of the normalized Laplacian. In this section, we will show how the ability to embed a metric space $(X, d)$ isometrically allows the concept of a geometric Fiedler vector to be extended to a geometric spectra for the graph with respect to the metric space $(X, d)$. For the sake of specificity, we will use the geometric Fiedler vector for the normalized Laplacian as defined by Radcliffe and Williamson [36] and Dumitriu and Radcliffe [10]. To that end, recall that given a connected graph $G=(V, E)$, the normalized Laplacian is defined as:

$$
\mathcal{L}=I-T^{-1 / 2} A T^{-1 / 2}
$$


where $A$ and $T$ are the adjacency and diagonal degree matrix of $G$, respectively. Specifically,

$$
\mathcal{L}_{x y}= \begin{cases}1 & \text { if } x=y \\ \frac{-1}{\sqrt{d_{x} d_{y}}} & \text { if }\{x, y\} \in E \\ 0 & \text { otherwise }\end{cases}
$$

where $d_{x}$ is the degree of vertex $x$ in the graph $G$. Now an eigenvector of $\mathcal{L}$ corresponding to the eigenvalue 0 is given by $T^{1 / 2} \mathbb{1}$ and thus by the Courant-Fisher Theorem,

$$
\lambda_{2}=\min _{x \perp T^{1 / 2} \mathbb{1}} \frac{\langle x, \mathcal{L} x\rangle}{\langle x, x\rangle} .
$$

Following the standard transformation in [9], we have that

$$
\frac{\langle x, \mathcal{L} x\rangle}{\langle x, x\rangle}=\frac{\left\langle x, T^{-1 / 2} L T^{-1 / 2} x\right\rangle}{\langle x, x\rangle}=\frac{\left\langle T^{-1 / 2} x, L T^{-1 / 2} x\right\rangle}{\langle x, x\rangle}=\frac{\langle f, L f\rangle}{\left\langle T^{1 / 2} f, T^{1 / 2} f\right\rangle}=\frac{\sum_{u \sim v}(f(u)-f(v))^{2}}{\sum_{v} f(v)^{2} d_{v}},
$$

where $f=T^{-1 / 2} x$. We will follow the terminology of [9] and refer to $f$ as a harmonic eigenvector for $\mathcal{L}$. It is worth noting that the harmonic eigenvectors generate the same spectrum as the standard eigenvectors. In particular,

$$
\lambda_{2}=\min _{f \perp T \mathbb{1}} \frac{\sum_{u \sim v}(f(u)-f(v))^{2}}{\sum_{v} f(v)^{2} d_{v}} .
$$

Furthermore, following the derivation in [9] the restriction on $f$ can be relaxed:

$$
\begin{aligned}
\lambda_{2}=\min _{f \perp T \mathbb{1}} \frac{\sum_{u \sim v}(f(u)-f(v))^{2}}{\sum_{v} f(v)^{2} d_{v}} & =\min _{f} \frac{\sum_{u \sim v}(f(u)-f(v))^{2}}{\sum_{v}(f(v)-\bar{f})^{2} d_{v}} \\
& =\min _{f \text { non-constant }} \frac{\operatorname{Vol}(G) \sum_{u \sim v}(f(u)-f(v))^{2}}{\sum_{u, v}(f(u)-f(v))^{2} d_{u} d_{v}},
\end{aligned}
$$

where $\bar{f}=\frac{\sum_{v} f(v) d_{v}}{\operatorname{Vol}(G)}$ and $\operatorname{Vol}(G)=\sum_{v} d_{v}$. From this formulation, the geometric Fiedler vector can be defined by noticing that $(f(u)-f(v))^{2}$ can be viewed as the squared distance between $f(u)$ and $f(v)$ on $\mathbb{R}$. Thus, the geometric Fiedler value of the normalized Laplacian over the metric $(X, d)$ is defined as:

$$
\lambda_{2}(G, X)=\min _{\substack{f: V \rightarrow X \\ f \text { non-constant }}} \frac{\operatorname{Vol}(G) \sum_{u \sim v} d(f(u), f(v))^{2}}{\sum_{u, v} d(f(u), f(v))^{2} d_{u} d_{v}} .
$$

Now suppose that $G$ can be isometrically embedded via $\phi$ into a Hilbert space $\mathcal{H}$ with kernel function $K$. Denote by $\|\cdot\|_{\mathcal{H}}$ and $\langle\cdot, \cdot\rangle_{\mathcal{H}}$ the norm and inner product on $\mathcal{H}$. For the sake of simplicity, we will assume that $\mathcal{H}=\operatorname{span}\{\phi(X)\}$. We then have that

$$
\lambda_{2}(G, X)=\min _{\substack{f \in \phi(X)^{|V|} \\ f \text { non-constant }}} \frac{\operatorname{Vol}(G) \sum_{u \sim v}\|f(u)-f(v)\|_{\mathcal{H}}^{2}}{\sum_{u, v}\|f(u)-f(v)\|_{\mathcal{H}}^{2} d_{u} d_{v}} .
$$

At this point, it would be natural to define orthogonality in terms of $\mathcal{H}$; however, we recall that $f \in \mathcal{H}$ is analogous to a harmonic eigenfunction of $\mathcal{L}$ over $\mathbb{R}$. Hence, it is necessary to invert the process of defining the harmonic eigenfunction in order to determine the appropriate notion of orthogonality. To that end, we first need the following multidimensional generalization of a standard algebraic fact. 
LEMMA 5.1. Let $\alpha_{1}, \ldots, \alpha_{n} \in \mathbb{R}^{k}$ and let $\alpha=\frac{1}{n} \sum_{i=1}^{n} \alpha_{i}$, then

$$
n \sum_{i=1}^{n}\left\|\alpha_{i}-\alpha\right\|^{2}=\sum_{1 \leq i<j \leq n}\left\|\alpha_{i}-\alpha_{j}\right\|^{2}
$$

Thus, we have that

$$
\lambda_{2}(G, X)=\min _{\substack{f \in \phi(X)^{|V|} \\ f \text { non-constant }}} \frac{\sum_{u \sim v}\|f(u)-\bar{f}+\bar{f}-f(v)\|_{\mathcal{H}}^{2}}{\sum_{u}\|f(u)-\bar{f}\|_{\mathcal{H}}^{2} d_{u}}
$$

where $\bar{f}=\frac{1}{\operatorname{Vol}(G)} \sum_{v} f(v) d_{v}$. Letting $g(u)=f(u)-\bar{f}$ and $P=\left\{f-\mathbb{1}_{|V|} \otimes \bar{f}: f \in \phi(X)^{|V|}\right\}$, this can be rewritten as:

$$
\begin{aligned}
\lambda_{2}(G, X) & =\min _{g \in P-\{0\}} \frac{\sum_{u \sim v}\|g(u)-g(v)\|_{\mathcal{H}}^{2}}{\sum_{u}\|g(u)\|_{\mathcal{H}} d_{u}} \\
& =\min _{g \in P-\{0\}} \frac{\sum_{u \sim v}\langle g(u)-g(v), g(u)-g(v)\rangle_{\mathcal{H}}}{\sum_{u}\langle g(u), g(u)\rangle_{\mathcal{H}} d_{u}} \\
& =\min _{g \in P-\{0\}} \frac{\sum_{u \sim v}\langle g(u)-g(v), g(u)-g(v)\rangle_{\mathcal{H}}}{\sum_{u}\left\langle\sqrt{d_{u}} g(u), \sqrt{d_{u}} g(u)\right\rangle_{\mathcal{H}}} \\
& =\min _{g \in P-\{0\}} \frac{\sum_{u \sim v}\langle g(u)-g(v), g(u)-g(v)\rangle_{\mathcal{H}}}{\left\langle T^{1 / 2} \otimes I_{\mathcal{H}} g, T^{1 / 2} \otimes I_{\mathcal{H}} g\right\rangle_{\mathcal{H}}|V|} \\
& =\min _{g \in P-\{0\}} \frac{\left\langle L^{1 / 2} \otimes I_{\mathcal{H}} g, L^{1 / 2} \otimes I_{\mathcal{H}} g\right\rangle_{\mathcal{H}}|V|}{\left\langle T^{1 / 2} \otimes I_{\mathcal{H}} g, T^{1 / 2} \otimes I_{\mathcal{H}} g\right\rangle_{\mathcal{H}}|V|},
\end{aligned}
$$

where the last equality follows from standard transformations of the combinatorial Laplacian combined with the fact that $L$ is positive semi-definite and has a matrix square root. Now, since $(X, d)$ is a finite metric space and $\mathcal{H}=\operatorname{span}\{\phi(X)\}$, we may think of $g$ as an element of $\mathbb{R}^{k|V|}$ for some finite $k$. Thus, we have

$$
\lambda_{2}(G, X)=\min _{\substack{g \in P \\ g \perp \operatorname{span}\left\{T \otimes e_{i}\right\}}} \frac{\left(L^{1 / 2} \otimes I_{k} g\right)^{T}\left(I_{|V|} \otimes K\right)\left(L^{1 / 2} \otimes I_{k} g\right)}{\left(T^{1 / 2} \otimes I_{k} g\right)^{T}\left(I_{|V|} \otimes K\right)\left(T^{1 / 2} \otimes I_{k} g\right)} .
$$

Since $K$ is a kernel function, both $K$ and $L$ are positive semi-definite and have unique matrix square roots. And so, letting $h=T^{1 / 2} \otimes K^{1 / 2} g$, we can see that the minimization can be re-expressed as a Rayleigh quotient in terms of $h$ for $\mathcal{L} \otimes I_{k}$. As a consequence, we see that two nonconstant functions $f_{1}: V \rightarrow X$ and $f_{2}: V: \rightarrow X$ should be considered orthogonal if and only if

$$
\begin{aligned}
0 & =\left\langle\left(T^{1 / 2} \otimes K^{1 / 2}\right)\left(f_{1}-\overline{f_{1}}\right),\left(T^{1 / 2} \otimes K^{1 / 2}\right)\left(f_{2}-\overline{f_{2}}\right)\right\rangle \\
& =\left\langle\left(T^{1 / 2} \otimes I_{\mathcal{H}}\right)\left(f_{1}-\overline{f_{1}}\right),\left(T^{1 / 2} \otimes I_{\mathcal{H}}\right)\left(f_{2}-\overline{f_{2}}\right)\right\rangle_{\mathcal{H}^{|V|} \mid} \\
& =\sum_{v} d_{v}\left\langle f_{1}(v)-\overline{f_{1}}(v), f_{2}(v)-\overline{f_{2}}(v)\right\rangle_{\mathcal{H}}
\end{aligned}
$$




$$
\begin{aligned}
& =\sum_{v} d_{v}\left\langle f_{1}(v), f_{2}(v)\right\rangle_{\mathcal{H}}-d_{v}\left\langle\overline{f_{1}}(v), f_{2}(v)\right\rangle_{\mathcal{H}}-d_{v}\left\langle f_{1}(v), \overline{f_{2}}(v)\right\rangle_{\mathcal{H}}+d_{v}\left\langle\overline{f_{1}(v)}, \overline{f_{2}}(v)\right\rangle_{\mathcal{H}} \\
& =\sum_{v} d_{v}\left\langle f_{1}(v), f_{2}(v)\right\rangle_{\mathcal{H}}-2 \sum_{v, u} \frac{d_{v} d_{u}}{\operatorname{Vol}(G)}\left\langle f_{1}(u), f_{2}(v)\right\rangle_{\mathcal{H}}+\sum_{v, x, y} \frac{d_{v} d_{x} d_{y}}{\operatorname{Vol}(G)^{2}}\left\langle f_{1}(x), f_{2}(y)\right\rangle_{\mathcal{H}} \\
& =\sum_{v} d_{v}\left\langle f_{1}(v), f_{2}(v)\right\rangle_{\mathcal{H}}-\sum_{x, y} \frac{d_{x} d_{y}}{\operatorname{Vol}(G)}\left\langle f_{1}(x), f_{2}(y)\right\rangle_{\mathcal{H}} .
\end{aligned}
$$

Before continuing, we should observe that if we relax the discrete nature of the embedding, that is, remove the restriction that $g \in P$, we end up with the spectrum of the operator $\mathcal{L} \otimes I_{\mathcal{H}}$ and an eigen decomposition that can be readily recovered from the standard decomposition of $\mathcal{L}$. Thus, we insist on the discrete nature of the embedding and note that that if $\mathcal{H}, K$, and $\phi$ come from the Schoenberg construction for point $x_{0}$, then the orthogonality condition can be simplified further by observing that for any two points in $\phi(X)$ the inner product is explicitly given by the kernel. Specifically, let $\tilde{f}_{1}: V \rightarrow X$ and $\tilde{f}_{2}: V \rightarrow X$ be two nonconstant functions from $V$ to the metric space. They should be considered orthogonal if and only if

$$
\begin{aligned}
0= & \sum_{v} \frac{d_{v}}{2}\left(d\left(\tilde{f}_{1}(v), x_{0}\right)^{2}+d\left(\tilde{f}_{2}(v), x_{0}\right)^{2}-d\left(\tilde{f}_{1}(v), \tilde{f}_{2}(v)\right)^{2}\right) \\
& \quad-\sum_{u, v} \frac{d_{u} d_{v}}{2 \operatorname{Vol}(G)}\left(d\left(\tilde{f}_{1}(v), x_{0}\right)^{2}+d\left(\tilde{f}_{2}(u), x_{0}\right)^{2}-d\left(\tilde{f}_{1}(v), \tilde{f}_{2}(u)\right)\right)^{2} \\
= & \sum_{u, v} \frac{d_{u} d_{v}}{2 \operatorname{Vol}(G)} d\left(\tilde{f}_{1}(v), \tilde{f}_{2}(u)\right)^{2}-\sum_{v} \frac{d_{v}}{2} d\left(\tilde{f}_{1}(v), \tilde{f}_{2}(v)\right)^{2} .
\end{aligned}
$$

It is worth noting that this definition is independent of the choice of base point, $x_{0}$, and furthermore does not depend on the existence of a particular embedding of $(X, d)$ into $\mathcal{H}$. Thus, it also provides a plausible definition for orthogonality of mappings when $(X, d)$ is not isometrically embeddable in a Hilbert space.

Furthermore, if we consider let $(X, d)$ be the standard metric space on $\mathbb{R}$ and recall that the harmonic eigenvectors for $\mathcal{L}$ over $\mathbb{R}$ satisfy $f \perp T \mathbb{1}$, we have that the orthogonality condition is

$$
\begin{aligned}
0 & =\sum_{u, v} \frac{d_{u} d_{v}}{2 \operatorname{Vol}(G)}\left(\tilde{f}_{1}(v)-\tilde{f}_{2}(u)\right)^{2}-\sum_{v} \frac{d_{v}}{2}\left(\tilde{f}_{1}(v)-\tilde{f}_{2}(v)\right)^{2} \\
& =\sum_{u, v} \frac{d_{u} d_{v}}{2 \operatorname{Vol}(G)}\left(\tilde{f}_{1}(v)^{2}-2 \tilde{f}_{1}(v) \tilde{f}_{2}(u)+\tilde{f}_{2}(u)^{2}\right)-\sum_{v} \frac{d_{v}}{2}\left(\tilde{f}_{1}(v)^{2}-2 \tilde{f}_{1}(v) \tilde{f}_{2}(v)+\tilde{f}_{2}(v)^{2}\right) \\
& =\sum_{v} d_{v} \tilde{f}_{1}(v) \tilde{f}_{2}(v)-\sum_{u, v} \frac{d_{u} d_{v}}{\operatorname{Vol}(G)} \tilde{f}_{1}(v) \tilde{f}_{2}(u) \\
& =\sum_{v} d_{v} \tilde{f}_{1}(v) \tilde{f}_{2}(v),
\end{aligned}
$$

which is precisely the condition for orthogonality for harmonic eigenvectors.

\section{REFERENCES}

[1] N. Alon and V.D. Milman. $\lambda_{1}$, isoperimetric inequalities for graphs, and superconcentrators. J. Combin. Theory Ser. B, 38(1):73-88, 1985.

[2] C.J. Alpert, A.B. Kahng, and S.-Z. Yao. Spectral partitioning with multiple eigenvectors. Discrete Appl. Math., 90(13):3-26, 1999. 
[3] S. Arora, J.R. Lee, and A. Naor. Euclidean distortion and the sparsest cut. J. Amer. Math. Soc., 21(1):1-21, 2008.

[4] S. Arora, S. Rao, and U. Vazirani. Expander flows, geometric embeddings and graph partitioning. J. ACM, 56(2):Art. 5, 37, 2009.

[5] N. Biggs. Algebraic Graph Theory, Second edition. Cambridge Mathematical Library. Cambridge University Press, Cambridge, 1993.

[6] A.E. Brouwer and W.H. Haemers. Spectra of Graphs. Universitext. Springer, New York, 2012.

[7] S. Chawla, A. Gupta, and H. Räcke. Embeddings of negative-type metrics and an improved approximation to generalized sparsest cut. ACM Trans. Algorithms, 4(2):Art. 22, 18, 2008.

[8] J. Cheeger. A lower bound for the smallest eigenvalue of the Laplacian. In: Problems in Analysis (Papers Dedicated to Salomon Bochner, 1969), 195-199. Princeton Univ. Press, Princeton, N. J., 1970.

[9] F.R.K. Chung. Spectral Graph Theory, Vol. 92. CBMS Regional Conference Series in Mathematics. Published for the Conference Board of the Mathematical Sciences, Washington, DC, 1997.

[10] I. Dumitriu and M. Radcliffe. On expansion of $g_{n, d}$ with respect to $g_{m, d}$. arXiv:1506.02614, 2015.

[11] M. Fiedler. Algebraic connectivity of graphs. Czechoslovak Math. J., 23(98):298-305, 1973.

[12] M. Fiedler. An algebraic approach to connectivity of graphs. In: Recent Advances in Graph Theory (Proc. Second Czechoslovak Sympos., Prague, 1974), 193-196. Academia, Prague, 1975.

[13] M. Fiedler. Laplacian of graphs and algebraic connectivity. In: Combinatorics and Graph Theory (Warsaw, 1987 ), Vol. 25. Banach Center Publ., 57-70. PWN, Warsaw, 1989.

[14] C. Godsil and G. Royle. Algebraic Graph Theory, Vol. 207. Graduate Texts in Mathematics. Springer-Verlag, New York, 2001.

[15] J.E. Goodman and J. O'Rourke, editors. Handbook of Discrete and Computational Geometry, Second edition. Discrete Mathematics and its Applications (Boca Raton). Chapman \& Hall/CRC, Boca Raton, FL, 2004.

[16] R.L. Graham. On isometric embeddings of graphs. In: Progress in Graph Theory (Waterloo, Ont., 1982), 307-322. Academic Press, Toronto, ON, 1984.

[17] R.L. Graham and P.M. Winkler. Isometric embeddings of graphs. Proc. Nat. Acad. Sci. U.S.A., 81(22, , Phys. Sci.):72597260, 1984.

[18] R.L. Graham and P.M. Winkler. On isometric embeddings of graphs. Trans. Amer. Math. Soc., 288(2):527-536, 1985.

[19] R.L. Graham and P.M. Winkler. Corrigendum to: 'On isometric embeddings of graphs' [Trans. Amer. Math. Soc. 288(2):527-536, 1985; MR0776391 (86f:05055b)]. Trans. Amer. Math. Soc., 294(1):379, 1986.

[20] L. Hagen and A.B. Kahng. New spectral methods for ratio cut partitioning and clustering. Trans. Comp.-Aided Des. Integ. Cir. Sys., 11(9):1074-1085, November 2006.

[21] P. Hjorth, P. Lisonĕk, S. Markvorsen, and C. Thomassen. Finite metric spaces of strictly negative type. Linear Algebra Appl., 270:255-273, 1998.

[22] P. Józiak. Conditionally strictly negative definite kernels. Linear Multilinear Algebra, 63(12):2406-2418, 2015.

[23] R. Krauthgamer, J.R. Lee, M. Mendel, and A. Naor. Measured descent: A new embedding method for finite metrics. Geom. Funct. Anal., 15(4):839-858, 2005.

[24] T.C. Kwok, L.C. Lau, Y.T. Lee, S.O. Gharan, and L. Trevisan. Improved Cheeger's inequality: Analysis of spectral partitioning algorithms through higher order spectral gap. In STOC'13-Proceedings of the 2013 ACM Symposium on Theory of Computing, 11-20. ACM, New York, 2013.

[25] J.R. Lee. On distance scales, embeddings, and efficient relaxations of the cut cone. In: Proceedings of the Sixteenth Annual ACM-SIAM Symposium on Discrete Algorithms, 92-101. ACM, New York, 2005.

[26] J.R. Lee, S.O. Gharan, and L. Trevisan. Multiway spectral partitioning and higher-order Cheeger inequalities. J. ACM, 61(6):Art. 37, 30, 2014.

[27] J.R. Lee, S.O. Gharan, and L. Trevisan. Multi-way spectral partitioning and higher-order Cheeger inequalities. In: STOC'12-Proceedings of the 2012 ACM Symposium on Theory of Computing, 1117-1130. ACM, New York, 2012.

[28] M. Mendel and A. Naor. Expanders with respect to Hadamard spaces and random graphs [extended abstract]. In: ITCS'14-Proceedings of the 2014 Conference on Innovations in Theoretical Computer Science, 353-358. ACM, New York, 2014.

[29] M. Mendel and A. Naor. Nonlinear spectral calculus and super-expanders. Publ. Math. Inst. Hautes Études Sci., 119:1-95, 2014.

[30] M. Mendel and A. Naor. Expanders with respect to Hadamard spaces and random graphs. Duke Math. J., 164(8):1471$1548,2015$.

[31] B. Mohar. Isoperimetric numbers of graphs. J. Combin. Theory Ser. B, 47(3):274-291, 1989.

[32] B. Mohar. The Laplacian spectrum of graphs. In: Graph Theory, Combinatorics, and Applications. Vol. 2 (Kalamazoo, MI, 1988), Wiley-Intersci. Publ., 871-898. Wiley, New York, 1991. 
[33] A.Y. Ng, M.I. Jordan, and Y. Weiss. On spectral clustering: Analysis and an algorithm. In: Proceedings of the 14th International Conference on Neural Information Processing Systems: Natural and Synthetic, NIPS'01, 849-856, Cambridge, MA, USA, 2001. MIT Press.

[34] V.I. Paulsen and M. Raghupathi. An Introduction to the Theory of Reproducing Kernel Hilbert Spaces, Vol. 152. Cambridge Studies in Advanced Mathematics. Cambridge University Press, Cambridge, 2016.

[35] A. Pothen, H.D. Simon, and K.-P. Liou. Partitioning sparse matrices with eigenvectors of graphs. SIAM J. Matrix Anal. Appl., 11(3):430-452, 1990. Sparse matrices (Gleneden Beach, OR, 1989).

[36] M. Radcliffe and C. Williamson. Bounds on geometric eigenvalues of graphs. arXiv:1501.03436, 2015.

[37] I.J. Schoenberg. On certain metric spaces arising from Euclidean spaces by a change of metric and their imbedding in Hilbert space. Ann. Math. (2), 38(4):787-793, 1937.

[38] I.J. Schoenberg. Metric spaces and positive definite functions. Trans. Amer. Math. Soc., 44(3):522-536, 1938.

[39] J. Shi and J. Malik. Normalized cuts and image segmentation. IEEE Trans. Pattern Anal. Mach. Intell., 22(8):888-905, August 2000.

[40] D.A. Spielman and S.-H. Teng. Spectral partitioning works: planar graphs and finite element meshes. In: 37th Annual Symposium on Foundations of Computer Science (Burlington, VT, 1996), 96-105. IEEE Comput. Soc. Press, Los Alamitos, CA, 1996.

[41] D.A. Spielman and S.-H. Teng. Spectral partitioning works: planar graphs and finite element meshes. Linear Algebra Appl., 421(2-3):284-305, 2007.

[42] J.C. Urschel and L.T. Zikatanov. On the maximal error of spectral approximation of graph bisection. Linear Multilinear Algebra, 64(10):1972-1979, 2016.

[43] P.M. Winkler. On graphs which are metric spaces of negative type. In Graph Theory with Applications to Algorithms and Computer Science (Kalamazoo, Mich., 1984), Wiley-Intersci. Publ., 801-810. Wiley, New York, 1985. 